\title{
Waterhouse-Friderichsen syndrome caused by a DF-2 bacterium in a splenectomised patient
}

\author{
AK CHAUDHURI,* RB HARTLEY, $\dagger$ AND AC MADDOCKS* \\ From the *Department of Bacteriology, Wright-Fleming Institute, St Mary's Hospital Medical School, \\ Paddington, London W2 1PG, and the †Department of Histopathology, St Mary's Hospital, Paddington, \\ London W2 1PG, UK
}

SUMMARY A case of Waterhouse-Friderichsen syndrome associated with septicaemia caused by a DF-2 group bacterium (dysgonic fermenter-type 2-Atlanta classification) is presented. Attention is drawn to the relation of DF-2 group septicaemias to dog bites (or contact) and impaired host defence after splenectomy.

\section{Case history}

A 57-year-old Caucasian woman developed abdominal pain and vomiting while on a weekend trip to London. After 6 hours she presented in the casualty department nauseated, dehydrated, and slightly disorientated but not critically ill. There was no history of previous illness apart from an operation for peptic ulcer in 1972 and slight dysuria for two weeks. On examination there were no abnormal findings. Blood cultures were taken, and she was admitted for rehydration, antibiotics, and observation with a diagnosis of possible cholecystitis or pyelonephritis. At this time her temperature was $39^{\circ} \mathrm{C}$, pulse $140 / \mathrm{min}$, BP $95 / 70 \mathrm{~mm} \mathrm{Hg}$, and white blood cells 13.4 $\left(\times 10^{9} / 1\right)$. Urea and electrolytes and midstream urine were normal.

The patient's condition gradually deteriorated despite antibiotic treatment initiated with ampicillin (1 g 6-hourly intravenously) to which was added penicillin (500 000 units intravenously 4-hourly) and metronidazole (500 mg intravenously 8-hourly). After 8 hours she developed petechial haemorrhages. After a further $3 \frac{1}{2}$ hours her WBC had fallen to $3 \cdot 6$ $\left(\times 10^{9} / 1\right)$, and clotting studies suggested disseminated intravascular coagulation. She became shocked and anuric and developed ecchymoses which became almost confluent. Cerebrospinal fluid taken at this time showed no abnormality.

In spite of supportive measures (blood transfusion, ventilation, and steroids) she died about 18 hours after admission.

Accepted for publication 23 June 1980
POST-MORTEM FINDINGS

A classical picture of Waterhouse-Friderichsen syndrome with ecchymoses and gross haemorrhage into the adrenals was seen. No abnormality was found at the old operation site; the spleen was absent. No focus of infection was found, and no organisms were seen in tissues stained with Dieterle's or Gram stains. There was no evidence of intravascular coagulation.

\section{BACTERIOLOGY}

Although Waterhouse-Friderichsen syndrome is associated with a variety of bacterial and viral infections, meningococcal infection is the commonest cause of the syndrome in our community, and blood cultures and cerebrospinal fluid were examined for Neisseria meningitidis. (The fact that the patient had no spleen was discovered only post mortem.)

The CSF culture was negative but the glucose broth blood culture was already slightly turbid at 20 hours, and vigorous efforts were made to find an organism. A Gram stain of the glucose broth showed weakly staining Gram-negative rods with tapered ends. Standard subculture to blood agar (Difco) incubated anaerobically (Gas Pak) and to serum chocolate agar (Difco) in $7 \% \mathrm{CO}_{2}$ gave no growth at 48 hours, but growth was obtained on non-selective Ison and Glynn ${ }^{1}$ neisseria medium in $7 \% \mathrm{CO}_{2}$ at 72 hours and on the serum chocolate agar at four days. Growth was later demonstrated on legionella medium supplied by Dr AG Taylor (Colindale) and on Difco Charcoal Pertussis agar. There was no growth on MacConkey agar. The organism's characteristics (Table) were those of a fastidious Gram-negative 
Some characteristics of the DF-2 group of fastidious Gram-negative rods

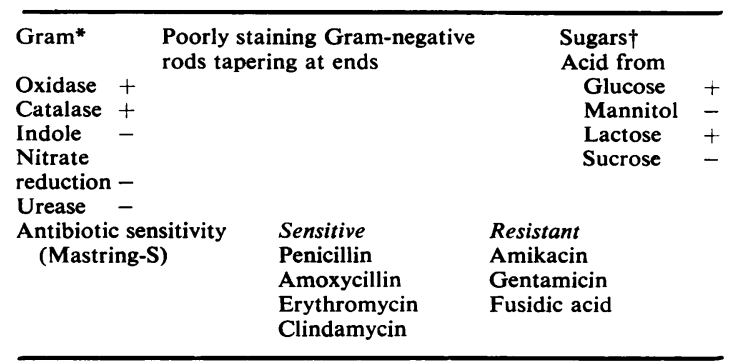

* Better visualised with carbol fuchsin.

+Dr Weaver used peptone/beef extract broth base with petrolatum seal to demonstrate fermentation in this group. ${ }^{2}$ There is no growth in peptone water sugars or $\mathrm{O} / \mathrm{F}$ medium, and growth but no reaction in serum water sugars without a seal.

rod, in some ways resembling Legionella pneumophila. The organism was sent for further investigation to the National Type Culture Collection and then to Dr Robert Weaver in Atlanta, Ga, USA. Dr Weaver was able to demonstrate that it belonged to the DF-2 (dysgonic fermenter) group of organisms which have been described by the Special Bacteriology Section, Communicable Disease Center, Atlanta, from 18 cases $^{2} 3$ (Table). There is considerable variation in the difficulty experienced in subculturing these organisms, and this was obviously one of the more 'difficult' ones.

\section{Discussion}

Relevant features of the DF-2 group are the association with dog bites (10/18 reported cases) or contact (5/18 cases) and impaired host defence, particularly after splenectomy (5/18 reported cases). The organisms have also been identified among dog oral flora by Bailie et al. ${ }^{4}$ Our patient had no spleen and was a dog owner.

The severe illness and rapid death in this case are the most dramatic so far described, but four deaths occurred among the 18 reported cases, where the splenectomised patients exhibited the most florid pictures, including meningitis and purpura.

The splenectomised patient has impaired defences, and a Waterhouse-Friderichsen picture can be induced by organisms that are found in the body flora and only rarely produce lesions. It is interesting to compare this case with the fatal Streptococcus milleri infection described by McCulloch and Parker: ${ }^{5}$ a member of the human oral flora produced a similar outcome in a splenectomised man. The comments in the British Medical Journal leading article 'After splenectomy' 6 on the possible use of spleen autotransplantation to prevent fatal septi- caemias are of interest in the light of these two cases. It should also be noted that the only evidence of splenectomy may be a history of peptic ulcer operation.

In spite of the preponderance of patients with impaired defences in the recorded cases with DF-2 infection it should be noted that two of the patients were apparently in good health and that infection was associated with cellulitis in some of the cases described by Butler $e t$ al. On the available evidence it is difficult to differentiate between the possibilities (a) that the organism is one of low pathogenicity able to cause septicaemia only in patients with poor immune response, and $(b)$ that the organism is more fastidious than other organisms commonly found in dog bite wounds, and its presence will be revealed only if carefully searched out.

The isolation of an 'unknown' organism raised some interesting problems, one being its similarity to L. pneumophila on initial investigation. The organism was tested by Dr AG Taylor (Colindale) against the available legionella antisera with negative results. The gas liquid chromatogram was done by $\mathrm{Dr}$ MJ Hudson (Colindale) for comparison with legionella and showed a definitively different picture from the characteristic legionella profile. This technique appears to have a very useful role in the early identification or exclusion of possible legionellas.

We thank Dr Robert E Weaver, of the Special Bacteriology Section, Communicable Disease Center, Atlanta, for his help in identifying this organism and Dr AG Taylor, Dr MJ Hudson, and Dr LR Hill, of Colindale, for their assistance.

We also thank Dr JG Walker, consultant physician at St Mary's Hospital, for permission to publish this case.

\section{References}

${ }^{1}$ Ison C, Glynn AA. Bulk growth of Neisseria gonorrhoeae type 1 in a biphasic system. Br J Vener Dis 1978;54:394-7.

${ }^{2}$ Butler T, Weaver RE, Venkata Ramani TK, et al. Unidentified gram-negative rod infection-a new disease of man. Ann Intern Med 1977;86:1-5.

${ }^{3}$ Schlossberg D. Septicaemia caused by DF-2. J Clin Microbiol 1978;9:297-8.

4 Bailie WE, Stowe EC, Schmitt AM. Aerobic bacterial flora of oral and nasal fluids of canines with reference to bacteria associated with bites. J Clin Microbiol 1978;7: 223-31.

${ }^{5}$ McCulloch DK, Parker A. Overwhelming post-splenectomy infection caused by Streptococcus milleri. J Infect Dis $1979 ; 1: 379-81$.

'Leading article "After splenectomy". Br Med J 1978;2: 1042-3.

Requests for reprints to: Dr AC Maddocks, Department of Bacteriology, Wright-Fleming Institute, St Mary's Hospital Medical School, London W2 1PG. 